\title{
On contractive projections in Hardy spaces
}

\author{
by \\ Florence Lancien (Besançon), \\ Beata Randrianantoanina (Oxford, OH) \\ and ERIC RicARD (Besançon)
}

\begin{abstract}
We prove a conjecture of Wojtaszczyk that for $1 \leq p<\infty, p \neq 2, H_{p}(\mathbb{T})$ does not admit any norm one projections with dimension of the range finite and greater than 1. This implies in particular that for $1 \leq p<\infty, p \neq 2, H_{p}$ does not admit a Schauder basis with constant one.
\end{abstract}

1. Introduction. The study of norm one projections and their ranges (1-complemented subspaces) has been an important topic of the isometric Banach space theory since the inception of the field. Contractive projections were also investigated from the approximation theory point of view, as part of the study of minimal projections, i.e. projections onto a given subspace with the smallest possible norm (cf. $[4,10])$. They are also closely related to the metric projections or nearest point mappings, and they are a natural extension of the notion of orthogonal projections from Hilbert spaces to general Banach spaces. Despite a great amount of work on contractive projections in various function spaces (cf. the survey [12]), very little is known about them in spaces of analytic functions.

To the best of our knowledge, the only results about contractively complemented subspaces of spaces of analytic functions are due to Wojtaszczyk [15], who proved that $H_{\infty}$ and the disc algebra $\mathcal{A}$ have "few" contractively complemented finite-dimensional subspaces.

Theorem 1.1 ([15, Theorem 4.3]). (a) If $P: \mathcal{A} \rightarrow \mathcal{A}$ is a norm one projection with finite-dimensional range of dimension greater than one, then $\operatorname{Im} P^{*}$ is contained in the set of measures $\mu$ which are singular with respect to the Lebesgue measure $\lambda$.

(b) If $P: H_{\infty} \rightarrow H_{\infty}$ is a norm one projection with finite-dimensional range of dimension greater than one, then $\operatorname{Im} P^{*}$ is contained in the space

2000 Mathematics Subject Classification: 46E15, 30D55, 46B20, $46 \mathrm{~B} 04$.

B. Randrianantoanina was a participant in NSF Workshop in Linear Analysis and Probability, Texas A\&M University. 
of functionals on $H^{\infty}$ such that every norm preserving extension to $L_{\infty}(\lambda)$ is singular with respect to the Lebesgue measure $\lambda$.

In particular it follows that the space $L_{1}(\lambda) / H_{1}^{0}(\lambda)$ whose dual is $H_{\infty}$ does not admit any norm one projection with $1<\operatorname{dim} \operatorname{Im} P<\infty$ [15, Corollary 4.4], and that $\mathcal{A}$ does not have a monotone basis $[15$, Theorem 4.6].

Wojtaszczyk asked about the situation in $H_{p}$ for $1 \leq p<\infty, p \neq 2$. Namely, he asked ([17] for $p=1,[14]$ for $p>1$ ) whether there exist any norm one projections on $H_{p}$ with $1<\operatorname{dim} \operatorname{Im} P<\infty$. He conjectured that the answer is no, which would imply in particular that $H_{p}$ does not admit a 1-unconditional basis, extending the result of [16] that $H_{1}$ does not admit a 1-unconditional basis.

In this paper we prove that Wojtaszczyk's conjecture is correct, i.e. that indeed, for $1 \leq p<\infty$ and $p \neq 2, H_{p}$ does not admit any norm one projections with $1<\operatorname{dim} \operatorname{Im} P<\infty$. In Section 4 we note that $H_{p}\left(\mathbb{T}^{2}\right)$ does have norm one projections with nontrivial finite-dimensional range and we include some remarks about the form of norm one projections on $H_{p}$ with infinite-dimensional range.

We note that it follows from an earlier work of Neuwirth [9] that $H_{p}$ does not admit a 1-unconditional basis for $1 \leq p<\infty, p \neq 2$. Actually, Theorem 7.5 therein shows that $H_{p}$ does not have the unconditional metric approximation property, a notion weaker than the existence of a 1-unconditional basis.

Acknowledgments. The research on this paper started during a sabbatical visit of the second named author at the Département de Mathématiques, Université de Franche-Comté in Besançon, France. She wishes to thank the Department and all members of the Functional Analysis Group for their hospitality during that visit.

We thank the referee for valuable comments.

\section{Preliminaries}

Definition 2.1. Let $X$ be a Banach space. We define the duality map $J$ from $X$ into subsets of $X^{*}$ by the condition that $f \in J(x) \subset X^{*}$ if and only if $\|f\|_{X^{*}}=\|x\|_{X}$ and $\langle f, x\rangle=\|x\|_{X}^{2}$.

We note that if $X$ is a strictly convex Banach space, then for all $x \in X$, the set $J(x)$ contains exactly one functional. In this case we will consider $J$ as a map from $X$ to $X^{*}$. If, in addition, $X$ is reflexive and $X^{*}$ is strictly convex, then $J^{*}: X^{*} \rightarrow X$, and $J^{*}=J^{-1}$.

Calvert [3] proved an important characterization of contractively complemented subspaces of reflexive Banach spaces in terms of the duality map $J$. 
TheORem $2.2([3])$. Let $X$ be a strictly convex reflexive Banach space with strictly convex dual. Then a closed linear subspace $Y$ of $X$ is 1-complemented in $X$ if and only if $J(Y)$ is a linear subspace of $X^{*}$.

This theorem is one of the most important tools in the theory of norm one projections. It has been proven independently also in [1] (using different methods).

Actually, we will need only a simple version of this last result (cf. [1, Proposition 1.1]):

Lemma 2.3. Let $X$ be a Banach space such that for any $x \in X, J(x)$ consists of exactly one point. Then, if $Y$ is finite-dimensional and 1-complemented in $X$, then $J(Y)$ linearly spans a finite-dimensional subspace of $X^{*}$ of dimension less than or equal to $\operatorname{dim} Y$.

Proof. Let $P$ be the contractive projection onto $Y$. For any $y \in Y$,

$$
\|y\|^{2}=\langle y, J(y)\rangle=\langle P(y), J(y)\rangle=\left\langle y, P^{*}(J(y))\right\rangle .
$$

But as $\left\|P^{*}(J(y))\right\| \leq\|J(y)\|$, using the hypothesis, we see that $P^{*}(J(y))=$ $J(y)$. So, $\operatorname{span} J(Y)$ is contained in the range of $P^{*}$.

We will need basic facts about duality in $H_{p}$, which we will recall now. More details can be found in [5] or [7].

By identifying each function in $H_{p}$ of the unit disk with the function defined by its boundary values on the torus, one can see $H_{p}$ as a subspace of $L_{p}(\mathbb{T})$ in the following way:

$$
H_{p}=H_{p}(\mathbb{T})=\left\{f \in L_{p}(\mathbb{T}): \widehat{f}(-n)=\int_{0}^{2 \pi} f\left(e^{i \theta}\right) e^{i n \theta} d \theta=0, \forall n \in \mathbb{N}^{*}\right\} .
$$

For $1 \leq p<\infty$ the dual of $H_{p}$ is isometrically isomorphic to the quotient $L_{q} / H_{q, 0}$, where $1 / p+1 / q=1$ and $H_{q, 0}=\left\{f \in H_{p}: f(0)=0\right\}$. More precisely, each functional $\phi$ in $H_{p}^{*}$ can be defined by a function $g$ in $L_{q}$ as follows: $\phi(f)=(2 \pi)^{-1} \int_{0}^{2 \pi} f\left(e^{i \theta}\right) g\left(e^{i \theta}\right) d \theta$ for $f \in H_{p}$, and two functions $g$ and $h$ in $L_{q}$ represent the same functional if and only if $g-h$ is in $H_{q, 0}$. This very elementary fact will be used in what follows.

Lemma 2.4. For $1 \leq p<\infty$ and each $f \in H_{p}$, there exists a unique element $J(f)$ in $H_{p}^{*}$ such that $\|J(f)\|_{H_{p}^{*}}=\|f\|_{H_{p}}$ and $\langle J(f), f\rangle=\|f\|_{H_{p}}^{2}$.

Proof. Since for $1<p<\infty, L_{p}$ spaces are strictly convex and uniformly smooth, $H_{p}$ as well as $H_{p}^{*}$ are strictly convex. This gives the result in the reflexive case.

For $p=1$, since $f$ is analytic and has full support, we know that there is a unique functional $g \in L_{\infty}$ such that $\|g\|_{L_{\infty}}=\|f\|_{H_{1}}$ and $\langle g, f\rangle=\|f\|_{H_{1}}^{2}$. Let $h \in J(f) \subset L_{\infty} / H_{\infty, 0}$. Then by Theorem 2 in [11], there is a lifting $\widetilde{h}$ 
of $h$ in $L_{\infty}$ such that $\|\widetilde{h}\|=\|h\|=\|f\|$. But then

$$
\langle\widetilde{h}, f\rangle=\langle h, f\rangle=\|f\|_{H_{1}}^{2} .
$$

So, we must have $\widetilde{h}=g$ and $h$ is unique.

Consequently, we can apply Lemma 2.3 to $H_{p}$ spaces for $1 \leq p<\infty$.

We point out that the map $J: H_{p} \rightarrow H_{p}^{*}$ is nonlinear but satisfies $J(a f)=|a| J(f)$ if $a \in \mathbb{C}$ and $f \in H_{p}$. We note that for $f \in H_{p}$, the function

$$
|f|^{p-2} \bar{f}=|f|^{p-1} \operatorname{sgn} \bar{f}
$$

where $\operatorname{sgn}\left(r e^{i \theta}\right)=e^{i \theta}$, is, up to a multiplicative constant, a representative of $J(f)$ in $H_{p}^{*}$.

\section{Main results}

Theorem 3.1. Let $1 \leq p<\infty, p \neq 2$. Then the Hardy space $H_{p}$ contains no 1-complemented subspaces of finite dimension larger than one.

Corollary 3.2. Let $1 \leq p<\infty, p \neq 2$. Then the Hardy space $H_{p}$ does not have a Schauder basis with constant 1 .

Proof of Theorem 3.1. Suppose for contradiction that there exists a finite-dimensional 1-complemented subspace $Y$ in $H_{p}$ with $\operatorname{dim} Y=d \geq 2$. There will be two different arguments depending on whether $p$ is an even integer or not.

(a) $p$ is not an even integer. Let $f_{1}, \ldots, f_{d}$ be linearly independent functions in $H_{p}$ such that $Y=\operatorname{span}\left\{f_{1}, \ldots, f_{d}\right\}$. By the preceding remarks, for each $f \in Y,|f|^{p-2} \bar{f}=|f|^{p} / f$ is a representative of an element of $J(Y)$. Thus

$$
J(Y)=\left\{\frac{|f|^{p}}{f} \oplus H_{q, 0}: f \in Y\right\}=\left\{\frac{\left|\sum_{i=1}^{d} \alpha_{i} f_{i}\right|^{p}}{\sum_{i=1}^{d} \alpha_{i} f_{i}} \oplus H_{q, 0}: \alpha_{i} \in \mathbb{C}, i=1, \ldots, d\right\} .
$$

Since $Y$ is 1-complemented in $H_{p}$ with $\operatorname{dim} Y=d \geq 2$, by Lemma $2.3, J(Y)$ is a linear subspace of $L_{q} / H_{q, 0}$. We define the following complex vector subspace of $L_{1} / H_{1,0}$ :

$$
Z=\operatorname{span}_{\mathbb{C}}\left\{\frac{|f|^{p}}{f} f_{i} \oplus H_{1,0}: f \in Y, i=1, \ldots, d\right\} .
$$

Since, by Lemma 2.3, $\operatorname{dim} J(Y) \leq d$, we conclude that the space $Z$ has dimension at most $d^{2}$ as a complex vector space and at most $2 d^{2}$ as a real vector space.

We now consider the two functions $f_{1}$ and $f_{2}$ which are linearly independent by assumption and we define $W$ and $W^{\prime}$ as the following real vector 
spans:

$$
\begin{aligned}
W^{\prime} & =\operatorname{span}_{\mathbb{R}}\left\{\left|\alpha_{1} f_{1}+\alpha_{2} f_{2}\right|^{p} \oplus H_{1,0}: \alpha_{1}, \alpha_{2} \in \mathbb{C}\right\} \subset L_{1} / H_{1,0}, \\
W & =\operatorname{span}_{\mathbb{R}}\left\{\left|\alpha_{1} f_{1}+\alpha_{2} f_{2}\right|^{p}: \alpha_{1}, \alpha_{2} \in \mathbb{C}\right\} \subset L_{1} .
\end{aligned}
$$

$W^{\prime}$ is contained in $Z$ : indeed, if $f=\sum_{i=1}^{2} \alpha_{i} f_{i}$, then $|f|^{p}=\sum_{j=1}^{2} \alpha_{j}\left(|f|^{p} / f\right) f_{j}$ is a representative of an element in $Z$. So $W^{\prime}$ is finite-dimensional. In turn, this implies that $W$ also has finite dimension. Indeed, there is a natural map $i: W \rightarrow W^{\prime}$ which sends a function to its equivalence class, and it is injective. This follows from the fact that we are dealing with real functions; a function $f$ in the kernel of $i$ is in $H_{1,0}$, but then $f$ is analytic and real, so $f$ is constant with null average, which means that $f=0$.

We next choose a subset $\Omega$ of the torus, of nonzero measure, such that there exists $a>0$ with $a<\left|f_{i}\right|<1 / a$ on $\Omega$ for $i=1,2$, and we define

$$
\widetilde{W}=\left\{\left.\frac{1}{\left|f_{1}\right|^{p}} h\right|_{\Omega}: h \in W\right\} .
$$

Just as $Y, Z$ and $W$, this space is of finite dimension. We are going to show that this leads to a contradiction.

For this purpose we consider the function $\Psi: \mathbb{C} \rightarrow \widetilde{W}$ defined by $\Psi(z)=$ $\left.\left|1+z\left(f_{2} / f_{1}\right)\right|_{\Omega}\right|^{p}$. Write $p=2 s$. Since $f_{2} / f_{1}$ is bounded and bounded away from zero on $\Omega$, we have the following power expansion for $\Psi$ valid for $|z|$ small enough (below all expressions $f_{2} / f_{1}$ are considered restricted to $\Omega$ ):

$$
\left|1+z \frac{f_{2}}{f_{1}}\right|^{p}=\left|\left(1+z \frac{f_{2}}{f_{1}}\right)^{s}\right|^{2}=\sum_{k, l \in \mathbb{N}}\left(\begin{array}{l}
s \\
k
\end{array}\right)\left(\begin{array}{l}
s \\
l
\end{array}\right)\left[\frac{f_{2}}{f_{1}}\right]^{k} \overline{\left[\frac{f_{2}}{f_{1}}\right]^{l}} z^{k} \bar{z}^{l} .
$$

Write $z=r e^{i \theta}$ for $r \in \mathbb{R}$ small enough and integrate in $\theta \in[0,2 \pi]$. We obtain a function $\Phi: I \rightarrow \widetilde{W}$, defined in a neighbourhood of 0 , such that

$$
\Phi(r)=\sum_{k \geq 0}\left(\begin{array}{l}
s \\
k
\end{array}\right)^{2}\left|\frac{f_{2}}{f_{1}}\right|^{2 k} r^{2 k}
$$

As $\Phi$ takes its values in $\widetilde{W}$, all coefficients in its power expansion are also in $\widetilde{W}$. Since $p$ is not an even integer, the binomial coefficient $\left(\begin{array}{l}s \\ k\end{array}\right)$ is never zero. One deduces that $\left|f_{2} / f_{1}\right|^{2 k}$ belongs to $\widetilde{W}$ for all $k \in \mathbb{N}$.

Since $\widetilde{W}$ is a finite-dimensional real vector space, there must exist $K \in \mathbb{N}$ and real coefficients $\alpha_{1}, \ldots, \alpha_{K}$, not all zero, such that

$$
\sum_{k=1}^{K} \alpha_{k}\left|\frac{f_{2}}{f_{1}}\right|^{2 k}=0
$$

This implies that $\left|f_{2} / f_{1}\right|$ takes values in the finite set of roots of the polynomial $\sum_{k=1}^{K} \alpha_{k} X^{2 k}$. The set $\Omega$ being of nonzero measure, we conclude 
that there exists a subset $\Omega_{0}$ of $\Omega$ and a constant $C_{0}$ with $\mu\left(\Omega_{0}\right)>0$ and $\left|f_{2} / f_{1}\right|=C_{0}$ on $\Omega_{0}$.

If we take $\left(f_{1}, f_{1}+f_{2}\right)$ instead of $\left(f_{1}, f_{2}\right)$, we obtain similarly the existence of a subset $\Omega_{1}$ of $\Omega_{0}$ and a constant $C_{1}$ with $\mu\left(\Omega_{1}\right)>0$ and $\left|1+f_{2} / f_{1}\right|=C_{1}$ on $\Omega_{1}$. Finally, on $\Omega_{1}$ we have $\left|f_{2} / f_{1}\right|=C_{0}$ and $\left|1+f_{2} / f_{1}\right|=C_{1}$. Since on $\mathbb{C}$ the equations $|z|=c_{0}$ and $|1+z|=c_{1}$ define two circles, this shows that on $\Omega_{1}, f_{1} / f_{2}$ takes its values in their intersection which consists of at most two points. By analyticity this implies that $f_{1}=\lambda f_{2}$ on $\Omega_{1}$, since $\mu\left(\Omega_{1}\right) \neq 0$. Therefore it follows that $f_{1}$ and $f_{2}$ are linearly dependent, which contradicts the definition of $f_{1}$ and $f_{2}$.

REMARK 3.3. The referee has pointed out to us that, alternatively, one can use the Rudin-Plotkin Equimeasurability Theorem $[13,8]$ to show that finite-dimensionality of $W$ implies that $f_{1} / f_{2}$ assumes only a finite number of values. A sketch of an argument is as follows:

If $\operatorname{dim} W<\infty$, then also $\operatorname{dim} V<\infty$, where $V=\operatorname{span}\left\{\left.\left|1+z\left(f_{1} / f_{2}\right)\right|_{\Omega}\right|^{p}\right.$ : $z \in \mathbb{C}\}$. Then we consider the space $V$ in $L_{1}$ with respect to the measure $\left|f_{1}\right|^{p} d t$. Take any $h \in L_{\infty}=\left(L_{1}\right)^{*}$ which annihilates $V$ and write $h=$ $h_{+}-h_{-}$(decomposition into the positive and negative part). We get

$$
\int\left|1+z f_{1} / f_{2}\right|^{p}\left(h_{+}\left|f_{1}\right|^{p}\right) d t=\int\left|1+z f_{1} / f_{2}\right|^{p}\left(h_{-}\left|f_{1}\right|^{p}\right) d t
$$

for all complex $z$. From the Equimeasurability Theorem we deduce that $f_{1} / f_{2}$ is equimeasurable with respect to both densities in parentheses. This implies that we have $\int \Psi\left(f_{1} / f_{2}\right) h\left|f_{1}\right|^{p} d t=0$ for any Borel bounded function $\Psi$ on $\mathbb{C}$. Since this holds for any $h$ which annihilates $V$ we infer that $\Psi\left(f_{1} / f_{2}\right) \in V$ for each $\Psi$. But all those compositions can be finite-dimensional only if $f_{1} / f_{2}$ assumes only a finite number of values.

(b) $p$ is an even integer. We denote by $P$ a contractive projection on $H_{p}$ such that $Y$ is the range of $P$. For fixed $h \in Y$ and $\phi \in H_{p}$, we have for all $z \in \mathbb{C}$,

$$
\|h+z \phi\|_{p}^{p} \geq\|h+z P(\phi)\|_{p}^{p} .
$$

The function $\Psi$ defined by $\Psi(z)=\|h+z \phi\|_{p}^{p}-\|h+z P(\phi)\|_{p}^{p}$ is a polynomial in $z$ and $\bar{z}$ which satisfies $\Psi(0)=0$ and $\Psi(z) \geq 0$ for all $z$, thus $\Psi^{\prime}(0)=0$. This yields, after restricting $\Psi$ to real $z$ only, and differentiating with respect to $z$,

$$
\operatorname{Re}\left[\int_{\mathbb{T}}|h|^{p-2} \bar{h}(\phi-P(\phi))\right]=0 .
$$

Since this holds for any $h$ in $Y$, by multiplying $h$ by complex constants of modulus one we obtain

$$
\int_{\mathbb{T}}|h|^{p-2} \bar{h}(\phi-P(\phi))=0, \quad \forall h \in Y, \forall \phi \in H_{p} .
$$


We now pick two functions $f$ and $g$ in $Y$ and define $\Theta$ by

$$
\Theta\left(z, z^{\prime}\right)=\int_{\mathbb{T}}\left|z^{\prime} f+z g\right|^{p-2} \overline{\left(z^{\prime} f+z g\right)}(\phi-P(\phi)) .
$$

By (3.1) we have $\Theta\left(z^{\prime}, z\right)=0$ for all $z, z^{\prime} \in \mathbb{C}$. Moreover, since $p$ is an even integer, $\Theta$ is a polynomial in $z$ and $z^{\prime}$. If we expand this polynomial, the coefficient of $\bar{z}\left|z^{\prime}\right|^{p-2}$ gives

$$
\int_{\mathbb{T}}|f|^{p-2} \bar{g}(\phi-P(\phi))=0, \quad \forall \phi \in H_{p}
$$

Consequently, for any $f \in Y$,

$$
\int_{\mathbb{T}}|f|^{p-2} \bar{h}(\phi-P(\phi))=0, \quad \forall h \in Y, \forall \phi \in H_{p} .
$$

We now introduce the scalar product $\langle\cdot, \cdot\rangle_{f}$ defined on $H_{p}$ by

$$
\langle h, k\rangle_{f}=\int_{\mathbb{T}}|f|^{p-2} \bar{h} k .
$$

It is a scalar product since $f$ has full support as it is analytic. We denote by $H_{f}$ the space $H_{p}$ equipped with the scalar product $\langle\cdot, \cdot\rangle_{f}$ and by $I_{f}$ the canonical injection from $H_{p}$ to $H_{f}$. With these new notations, (3.2) can be written

$$
\langle h, \phi-P(\phi)\rangle_{f}=0, \quad \forall h \in Y, \forall \phi \in H_{p} .
$$

In particular for $h=P(\phi)$ we obtain

$$
\langle P(\phi), \phi-P(\phi)\rangle_{f}=0, \quad \forall \phi \in H_{p} .
$$

Thus

$$
\|P(\phi)\|_{f}^{2}=\langle\phi, P(\phi)\rangle_{f} \leq\|\phi\|_{f}\|P(\phi)\|_{f}, \quad \forall \phi \in H_{p} .
$$

This means that $P_{f}$ defined by $P(\phi)=P_{f}(\phi)$ on $H_{p}$ is an orthogonal projection on $H_{f}$ which coincides with $P$.

From now on, we consider two linearly independent functions $f$ and $g$ in $Y$. We have another scalar product $\langle\cdot, \cdot\rangle_{g}$, a space $H_{g}$ and an orthogonal projection $P_{g}$ on $H_{g}$. We next consider a basis $h_{1}, \ldots, h_{d}$ of $Y$ which is orthogonal simultaneously for $\langle\cdot, \cdot\rangle_{f}$ and $\langle\cdot, \cdot\rangle_{g}$; this is possible because $Y$ is assumed to be finite-dimensional. Indeed, if $\left(e_{i}\right)_{i=1}^{d}$ is a $\langle\cdot, \cdot\rangle_{f}$-orthonormal basis, and $A$ is the matrix of $\langle\cdot, \cdot\rangle_{g}$ in that basis, then $A$ is self-adjoint and positive, and thus there exists a $\langle\cdot, \cdot\rangle_{f}$-orthonormal basis which diagonalizes $A$, and thus is $\langle\cdot, \cdot\rangle_{g}$-orthogonal. Then, as $P_{f}$ and $P_{g}$ are orthogonal projections,

$$
P_{f}=\sum_{i=1}^{d} \frac{\left\langle\cdot, h_{i}\right\rangle_{f} h_{i}}{\left\|h_{i}\right\|_{f}}, \quad P_{g}=\sum_{i=1}^{d} \frac{\left\langle\cdot, h_{i}\right\rangle_{g} h_{i}}{\left\|h_{i}\right\|_{g}} .
$$


Since for all $\phi \in H_{p}$ we have $P_{f}(\phi)=P_{g}(\phi)=P(\phi)$, we obtain

$$
\frac{\left\langle\phi, h_{1}\right\rangle_{f} h_{1}}{\left\|h_{1}\right\|_{f}}=\frac{\left\langle\phi, h_{1}\right\rangle_{g} h_{1}}{\left\|h_{1}\right\|_{g}}, \quad \forall \phi \in H_{p}
$$

If we set $\alpha=1 /\left\|h_{1}\right\|_{f}$ and $\beta=1 /\left\|h_{1}\right\|_{g}$, this yields

$$
\int_{\mathbb{T}}\left(\alpha|f|^{p-2}-\beta|g|^{p-2}\right) \bar{h}_{1} \phi=0, \quad \forall \phi \in H_{p} .
$$

This means that $\left(\alpha|f|^{p-2}-\beta|g|^{p-2}\right) \bar{h}_{1}=0$ in $H_{p}^{*}$, thus there exists $h \in H_{q, 0}$ so that

$$
\left(\alpha|f|^{p-2}-\beta|g|^{p-2}\right)\left|h_{1}\right|^{2}=h h_{1} .
$$

Again the fact that the left hand side is real and the analyticity of the right hand side imply that they are both zero.

Finally, we obtain $|f|^{p-2}=\lambda|g|^{p-2}$ for some positive constant $\lambda$. This reasoning holds for any two functions $f$ and $g$ linearly independent in $Y$. If we take $f+g$ and $g$ instead of $f$ and $g$, we find that $|f+g|^{p-2}=\nu|g|^{p-2}$ for some positive constant $\nu$. But we have already explained that this cannot happen unless $f$ and $g$ are linearly dependent, which contradicts our hypothesis.

4. Comments. The above result is purely isometric. By that we mean that for any $d>1$ and $1 \leq p \leq \infty$ and $\varepsilon>0$, there are $d$-dimensional subspaces of $H_{p}$ which are $1+\varepsilon$-complemented. This is an easy consequence of the Szegö theorem as it provides functions in $H_{p}$ which have almost disjoint supports.

One can also wonder if Theorem 3.1 has extensions to domains other than the torus $\mathbb{T}$. As soon as there are more variables, the situation becomes quite different:

Proposition 4.1. For all $1 \leq p \leq \infty$ and any $d>1$, there are 1 complemented d-dimensional subspaces in $H_{p}\left(\mathbb{T}^{2}\right)$.

Proof. This is just a transference argument. Let $d>1$ be fixed and for each function $f\left(z_{1}, z_{2}\right)$ on $\mathbb{T}^{2}$, define

$$
T(f)\left(z_{1}, z_{2}\right)=\int_{T} f\left(z z_{1}, z z_{2}\right) \bar{z}^{d+1} d z .
$$

It is not hard to see that $T$, for any $p$, is a contractive projection from $L_{p}$ onto the subspace spanned by the functions $z_{1}^{k} z_{2}^{l}$ with $k+l=d-1$.

The same kind of argument carries over to $H_{p}\left(S_{n}\right)$ where $S_{n}$ is the unit sphere in $\mathbb{C}^{n+1}$, for any $n \geq 1$. As an easy consequence, $H_{p}(\mathbb{T})$ and $H_{p}\left(\mathbb{T}^{2}\right)$ are not isometric. 
We will now describe some infinite-dimensional 1-complemented subspaces of $H_{p}$. For $\Lambda$ a subset of $\mathbb{N}$, we define

$$
H_{p, \Lambda}=\left\{f \in H_{p}(\mathbb{T}): \widehat{f}(n)=0, \forall n \in \mathbb{N} \backslash \Lambda\right\} .
$$

It is well known that if $\Lambda$ is a coset in $\mathbb{N}$ then $H_{p, \Lambda}$ is 1 -complemented in $H_{p}$; in that case the projection $P: H_{p} \stackrel{\text { onto }}{\longrightarrow} H_{p, \Lambda}$ is the Fourier multiplier defined by the characteristic function of $\Lambda$.

More generally, for $\psi$ an inner function with $\psi(0)=0$, one can consider $H_{p}^{\psi}$, the closure in $H_{p}$ of the algebra generated by $\psi$. J. A. Ball [2] proved that there exists an expectation operator $P_{\psi}$ which is a norm one projection from $H_{p}$ onto $H_{p}^{\psi}$.

From this result we can deduce generalizations of the spaces $H_{\Lambda}$ above. For $\psi$ an inner function with $\psi(0)=0$ and $\Lambda=\{q k+r: n \in \mathbb{N}\}$ where $q \in \mathbb{N}$ and $r \in\{0, \ldots, k-1\}$, we can consider $H_{p, \Lambda}^{\psi}$, the closure in $H_{p}$ of the set $\left\{\psi^{n}: n \in \Lambda\right\}$. It is easy to see that $H_{p, \Lambda}^{\psi}$ is a 1 -complemented subspace of $H_{p}$ via the projection defined for $f \in H_{p}$ by $P_{\psi, \Lambda}(f)=\psi^{r-q} P_{\psi^{q}}\left(f \psi^{q-r}\right)$ if $r \neq 0$ and $P_{\psi, \Lambda}(f)=P_{\psi^{q}}(f)$ if $r=0$. (Note that $H_{p, \Lambda}$ corresponds to the case $\psi(z)=z$.)

Moreover, it is clear that if $X$ is any Banach space, $T$ is an isometry from $X$ onto $X$, and $P: X \stackrel{\text { onto }}{\longrightarrow} Y$ is a norm one projection, then $Q=$ $T P T^{-1}: X \stackrel{\text { onto }}{\longrightarrow} T(Y)$ is also a norm one projection, and thus $T(Y)$ is 1-complemented in $X$. Isometries of $H_{p}$ have been described by Forelli [6] who proved the following.

Theorem 4.2 ([6, Theorem 2]). Suppose that $p \neq 2$ and $T$ is a linear isometry of $H_{p}$ onto $H_{p}$. Then

$$
T f=b\left(\frac{d \phi}{d z}\right)^{1 / p} f(\phi),
$$

where $\phi$ is a conformal map of the unit disk onto itself and $b$ is a unimodular complex number. Conversely, (4.1) defines a linear isometry of $H_{p}$ onto $H_{p}$.

If we denote by $T_{\phi}$ an isometry of this form we obtain new norm one projections by composition with the preceding projections $P_{\psi, \Lambda}$ :

$$
Q=T_{\phi} P_{\psi, \Lambda} T_{\phi}^{-1}: H_{p} \stackrel{\text { onto }}{\longrightarrow} T_{\phi}\left(H_{p, \Lambda}^{\psi}\right)
$$

is also a contractive projection, and thus $T_{\phi}\left(H_{p, \Lambda}^{\psi}\right)$ is 1-complemented in $H_{p}$.

We do not know whether every 1-complemented subspace of $H_{p}$ has to be isometric to $H_{p, \Lambda}^{\psi}$ for some coset $\Lambda$ in $\mathbb{N}$ and some inner function $\psi$ vanishing at the origin. 


\section{References}

[1] J. Arazy and Y. Friedman, Contractive projections in $\mathcal{C}_{p}$, Mem. Amer. Math. Soc. 459 (1992).

[2] J. A. Ball, Hardy space expectation operators and reducing subspaces, Proc. Amer. Math. Soc. 47 (1975), 351-357.

[3] B. Calvert, Convergence sets in reflexive Banach spaces, Proc. Amer. Math. Soc. 47 (1975), 423-428.

[4] E. W. Cheney and K. H. Price, Minimal projections, in: Approximation Theory (Lancaster, 1969), Academic Press, London, 1970, 261-289.

[5] P. L. Duren, Theory of $H^{p}$ Spaces, Pure Appl. Math. 38, Academic Press, New York, 1970.

[6] F. Forelli, The isometries of $H^{p}$, Canad. J. Math. 16 (1964), 721-728.

[7] K. Hoffman, Banach Spaces of Analytic Functions, Prentice-Hall, Englewood Cliffs, NJ, 1962. (Reprinted by Dover Publ. in 1988.)

[8] A. Koldobsky and H. König, Aspects of the isometric theory of Banach spaces, in: Handbook of the Geometry of Banach Spaces, Vol. I, North-Holland, Amsterdam, 2001, 899-939.

[9] S. Neuwirth, Metric unconditionality and Fourier analysis, Studia Math. 131 (1998), $19-62$.

[10] W. Odyniec [V. P. Odinec] and G. Lewicki, Minimal Projections in Banach Spaces, Problems of Existence and Uniqueness and Their Application, Lecture Notes in Math. 1449, Springer, Berlin, 1990.

[11] L. B. Page, Bounded and compact vectorial Hankel operators, Trans. Amer. Math. Soc. 150 (1970), 529-539.

[12] B. Randrianantoanina, Norm one projections in Banach spaces, Taiwan. J. Math. 5 (2001), 35-95.

[13] W. Rudin, $L^{p}$-isometries and equimeasurability, Indiana Univ. Math. J. 25 (1976), 215-228.

[14] P. Wojtaszczyk, private communication.

[15] - On projections in spaces of bounded analytic functions with applications, Studia Math. 65 (1979), 147-173.

[16] - The Franklin system is an unconditional basis in $H_{1}$, Ark. Mat. 20 (1982), 293-300.

[17] —, Spaces of analytic functions with integral norm, in: Handbook of the Geometry of Banach Spaces, Vol. 2, North-Holland, Amsterdam, 2003, 1671-1702.

Département de Mathématiques

Université de Franche-Comté

16 Route de Gray

25030 Besançon, France

E-mail: Florence.Lancien@math.univ-fcomte.fr

Eric.Ricard@math.univ-fcomte.fr
Department of Mathematics and Statistics Miami University Oxford, OH 45056, U.S.A. E-mail: randrib@muohio.edu

Received January 11, 2005

Revised version April 14, 2005 